\title{
Algunos datos y consideraciones sobre el autoritarismo de la clase trabajadora
}

\author{
José R. Torregrosa
}

\section{Introducción}

La obra, ya clásica, de Adorno y sus colaboradores sobre "La Personalidad Autoritaria" (1) inició una de las líneas de investigación más fecundas de la Fisicología Social contemporánea. En dos monumentales volúmenes y con todo el aparato técnico de distintas ciencias sociales, se ponía de manifiesto cómo determinadas actitudes intolerantes, etnocéntricas y pre-fascistas se hallaban integradas en el núcleo mismo de la estructura y funcionamientc de la personalidad individual. Orientaciones de inmediata significación sccio-folítica podían interpretarse a partir de una gama reducida de variables psicológicas, cuya específica estructura servía -o podría servir - de sopcrte a movimientos e i.jeologías antidemocráticos. Perspectivas psicológico-históricas de gran significación, como la de un Frcmm, un Ranulf, por ejamplo, eran vertidas en un lenguaje más "operativo", más manejable desde el punto de vista de la investigación empírica. La escala "F", entre otras. aparecía ccmo producto de este esfuerzo. Es indudable que la gran proliferación de investigaciones que han proseguido en la línea de los autores de "La Personalidad Autoritaria" se ha debido, en no poca medida, a la disponibilidad de este instrumento de investigación.

Pero, precisamente por su alcance y significación, "La Personalidad Autoritaria" fue sometida desde su aparición a profundes análisis críticos, tanto desde el punto de vista teórico como metodológico. El volumen de Christie, R. y Jahoda, M. (2) sigue siendo, en este sentido, el mejor ejemplo. El trabajo de Hyman y Sheastsley (3), por una parte, y el del mismo Christie (4), por otra, contenidos en dicho volumen, independientemente de su valor

(1) Adorno, T. W.; Frenket-Brunswik, E,; Levinson, 1). J., y Sanford, R. N.: The Authoritarian Personality. Harper, New York, 1950. Traducción castellana: Ed. Proyección, Buenos Aires, 1965.

(2) Christie, R. y JahodA, M. (Eds.): Studies in the Scope and Method of "The Authoritarian Personality". The Free Press. Gleucoe, Ill., 1954.

(3) Hymax, H. H. y Sheatslex, P. B.: "The Authoritarian Personality: A Methodological Critique", en Richard Christie y Marie Jahoda (Eds.), op. cit., págs. 50-122.

(4) Christie, R.: "Authoritarianism Re-examined", en Christie y Jahoda (Eds.), op. cit. págs. $123-195$. 
metodológico, contienen matizaciones de gran valor teórico-sustantivo. Hyman y Sheatsley hacen notar que las altas puntuaciones en la escala " $F$ " bien pudieran deberse a diferencias de nivel intelectual, más bien que a una específica estructura de la personalidad; Christie cuestiona el supuesto del grupo de Berkeley de que la pertenencia y participación de los sujetos en distintos grupos saciales sea menos relevante para explicar sus personalidades autoritarias que los componentes profundos de su personalidad. Por su parte, Shils (5) observa que los autores de "La Personalidad Autoritaria" tratan sólo del autoritarismo de la derecha, pero que el autoritarismo puede ser también una característica de crientaciones izquierdistas. Es decir, no sćlo son autoritarias las personas de ideología fascista, sino también las personas de ideología ccmunista, y la escala "F" no es sensible al de estas últimas. En este sentido se sitúa la obra de Eysenk (6) al querer aislar los factores estructurales comunes de la mentalidad autoritaria, tanto de corte fascista como comunista.

En esta úitima línea se sitúan también las investigaciones de Rokeach (7) sobre la mentalidad "dcgmática". El dcgmatismo es un rasgo estructural de los sistemas cognitives independiente de los contenidos específicos de los mismos. Se puede ser dogmático en ciencia, en política, en religión, en cualquier esfera de experiencia, en definitiva. Se trata de un modo de organización de las creencias, más bien que del contenido de dichas creencias. La formulación de Rokeach pretende ser más amplia que la formulada en "La Personalidad Autoritaria", en el sentido de que abcrda el problema del astoritarismo general y no sólo el autoritarismo de la mentalidad conservadora y convencional, potencialmente fascista. La estructura de su teoría es más cognitiva que motivacional; es más psicología de la forma que psicoanálisis.

Fero independientemente de las críticas teóricas y metcdológicas, y de los intentes de superación, la construcción hipotética "autoritarismo", derivada, a su vez, de la de "personalidad autoritaria", ha sido un componente importante en el marco conceptual de muchas investigaciones empíricas, desde el campo de la percepción interpersonal hasta el de la sociología

(5) Shis., E. A.: Authoritarianism: "Right" and "Left", en Christie y Jahoda (Eds.), op. cit., pígs. 24-49.

(6) Eysenk, H. J.: Psicología de la decisión politica. Ariel, Barcelona, 1964.

(7) a) Roкеaсh, M.: "The Nature and Meaning of Dogmatism", en E. P. Hollander y R. G. Hunt (Eds.): Current Perspectives in Social Psychology. Oxford Univ. Press, New York, 1963. Págs. 162-172.

b) Rokfach, M.: The Open and Closed Mind. Basic Books. New York, 1960. 
política, desde la psicología individual a la psicología de clase (8); las definiciones operativas utilizadas en dichas investigaciones han sido la escala "F", original o modificada; la escala de dogmatismo de Rokeach parte de las mismas e instrumentos similares. De un síndrome cuyas conexiones e interdependencias con otras estructuras y mecanismos de la personalidad son especificadas (por medio de técnicas psicológicas complementarias con la escala "F") se ha pasado a las meras constataciones de aspectos aislados del síndrome autoritario, y, a partir de las mismas, a generalizaciones sobre la psicología política diferencial de distintas clases sociales.

De este modo, una formulación teórica que había sargido teniendo como referente real el comportamiento y las crientaciones de personas de una clase sccial específica - la burguesía y, en especial, la pequeña burguesíasobre la que se han apoyado, fundamentalmente, los fascismos y pre-fascismos contemporáneos, es ahora extendida —cambiando el énfasis de los aspectos motivacionales a los aspectos cognitivos- a otras clases sociales, sobre todo, a la clase trabajadora.

\section{Hipótesis}

En esta línea cabe situar el capítulo cuarto del libro de Lipset "El hombre político: Bases sociales de la política", dedicado al autoritarismo de la clase trabajadora (9). Lipset abre el capítulo con el siguiente texto: "La progresiva percatación de que los movimientos extremistas e intolerantes de la sociedad moderna están basades más en las clases bajas que en las medias y altas, ha puesto en un dilema a los intelectuales de la izquierda democrática que crejeron que el proletariado sería una fuerza para la libertad, la igualdad social y el progreso social" (10). Después de citar una entrevista con un joven obrero fascista inglés, que participó en actividades violentas contra los negros, Lipset perfila su planteamiento:

(8) Para una reciente panorámica de las investigaciones relacionadas con "La Personalidad Autoritaria" véase Kirscht, J. P. y Dit.enay, R. C.: Dimensions of Authoritarianism: A Review of Research and Theory. Univers. of Kentuky Press. Lexington, 1967.

(9) Lipset, S. M.: Political Man (1960). Anchor Books. 1963, págs. 87-126. En un amplio y bien documentado trabaio en el que se examina la hipótesis de Lipset, fundamentalmente a la luz de estudios electorales, Manuel Ramíkez Giménez concluye: "Tampoco los datos electorales para 1964 permiten una constatación importante del autoritarisino obrero. Sin duda habrá que esperar posteriores investigaciones sobre estos mismos datos para sacar conclusiones. Pero, al menos en los temas tratados, li clase trabajadora no sa muestra especialmente mís o menos liberal o más o menos autoritaria que la clase media o que la clase alta" "La clase trabajadora en Fstados Unidos", en Reviśa Española de la Opinión Pública, n. ${ }^{\circ}$ 5, 1966. Página 114.

(10) LiPSET, op. cit., paíg. 87. 
"Tan claras demcstraciones del etnccentrismo de la clase obrera y de su afo, c a los movimientos políticos totalitarios coinciden con estudios de cpinión pública, religión, sociología de la familia y estructura de la personalidad. Muchos de estos estudios sugieren que el estilo de vida de la clase cbrera produce individuos con perspectivas $\mathrm{y}$ orientaciones políticas rígidas e intolerantes" (11). Aunque Lipset reconoce que de las actitudes inferidas de datos de encuesta sólo no puede predecirse el apoyo que cierto estrato sacial puede prestar a movimientos autoritarios o democráticos, apunta sin embargo que "tanto la evidencia empírica como la teoría sugieren que las clases tajas son relativamente más autoritarias, que son más susceptibles de ser atraídas hacia movimientos extremistas que moderados o democráticcs..." (12). Desde este planteamiento, y apoyándose en una serie de investigaciones, Lipset prosigue su caracterización sociopsicológica de la clase trabajadora: su relativa mayor propensión al partido único que el pluripartidismo, a la "mentalidad dura", a la intolerancia; su mayor adaptación y confcrmismo; su mayor propensión a adherirse a movimientos religiosos extremistas; su menor nivel de educación formal; su menor nivel de participación en organizaciones formales; su menor exposición a la lectura de libros, revistas y feriódicos que las clases medias o altas y, en general, a participar menos en la vida política. Cada una de estas características está rełacionada negativamente con las actitudes democráticas. De igual modo, la falta de seguridad económica y psicológica aparece como un factor importante en la predisposición al autoritarismo de las clases trabajadoras. Por ctra parte, la estrechez de perspectivas, la excesiva preccupación por el hic et nunc, la mayor dificultad en llegar a abstracciones desde experiencias concretas, la falta de imaginación, la mayor sugestionalidad e incapacidad de percibir problemas complejos, su antiintelectualismo, su falta de perspectiva gradualista del cambio social... "Todas estas características forman parte de las complejas bases psicológicas del autoritarismo" (13).

¿En qué medida, cabe preguntarse, este planteamiento es aplicable en el contexto social español? ¿En qué medida los datos de que disponemos anoyan o no la hipótesis de Lipset? ¿En qué medida este planteamiento responde al planteamiento original de los autores de "La Personalidad Autoritaria"? ¿En qué medida el potencial fascista es mayor en la clase trabajadora que en las clases medias o altas?

(11) LipseT, op. cit., págs. 88-89.

(12) Lipset, op. cit., págs. 91-92.

(13) L.IPSET, op. cit., pág. 108. 


\section{Los datos}

Entre los datos que Lipset aporta para probar su hipótesis sobre el relativo mayor autoritarismo de las clases trabajadoras, algunos provienen de estudics por encuesta realizados en varios países. Pcr ello, los datos que vamos a presentar son, en principio, ré'evantes para la hifótesis planteada (14).

En nuestrc caso, utilizaremes ccmo indicadores de autoritarismo las raspuestas a items de la escala de dogmatismo de Rokeach y las respuestas a items de la escala "F" adaptada y utilizada por Pinillos (15). La escala de Rokeach intenta detectar una serie de dimensiones que caracterizan a los sistemas de creencias cerrados, es decir, dogmáticos. Ahora bien, y como afirma el propic Rokeach (16), la escala debe servir también para medir el autoritarismo general e intolerancia. Por otra parte, los items que utilizamos pertenecen específicamente a las subescalas, así denominadas por el propio Rckeach, además de otros items que intentan detectar la orientación temporal. Fueron ircluides en un cuestionario más amplio aplicado a una muestra nacional representativa de la población española de 15 a 40 años. Este estudio fue realizado por el Instituto de la Opinión Pública en colaboración con el Instituto de Investigación sobre la Paz, de Oslo.

Lcs items fueron introducidos a los entrevistados del siguiente modo: "A continuación podrá Vd. leer una serie de afirmaciones o juicios sobre ccsas distintas. (El entrevistador entrega al entrevistado una hoja con los items). Nos gustaría saber si Vd. está de acuerdo, en desacuerdo, o si se siente indeciso con respecto a cada una de esas afirmaciones. Por favor, dígame, a medida que los vayamos leyendo, si está Vd. de acuerdo, indeciso, o en desacuerdo". Los items utilizados, ordenados por el poder discriminatorio de los mismos, aparecen en el Cuadro I. A pesar de las diferencias cbservables en el "poder discriminatorio", totos los items han sido incluidos fara establecer la clasificación de los entrevistados en "autoritarismo alto" y "autoritarismo bajo" (17).

En el Cuadro II pueden cbservarse las relaciones entre las puntuaciones en los items de la escala de Rokeach y distintas variables socioeconómicas.

(14) Ramíkez Gimínz hace notar, en el trabajo ya citado, la carencia de datos para España, así como la necesidad de una perspectiva comparativa.

(15) Pinulos, J. L.: "Análisis de la escala "F" en una muestra española". Revista de Psicología General y Aplicada, n. ${ }^{\circ}$ 70, 1963. Págs. 115.5-1174.

(16) Op. cit. b) páig. 72.

(17) Para obtener las puntuaciones se asignó un 0 por la respuesta "en desacuerdo": un 1 por "indeciso" y un 2 por "de acuerdo". Dado que han sido utilizados 16 items, la puntuación puede oscilar entre 0 y 32. Los "baios" tienen una puntuación entre 0 y 18 y los "altos" entre 26 y 32, incluyendo aproximadamente un 25 por 110 de los casos cada uno de estos extremos de la distribución. 


\title{
CUADRO 1
}

\author{
Items incluides en la escala
}

P. D.

En un mundo tan complicado como el ạctual, la única forma de enterarse de lo que realmente pasa es atenerse a lo que nos digan los jefes y

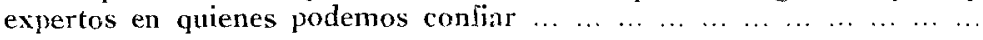

Llegar a un compromiso con nuestros oponentes resulta peligroso porque, en muchos casos, nos lleva a traicionar a nuestro propio bando...

El presente está lleno de desgracias e infelicidades casi siempre; lo que

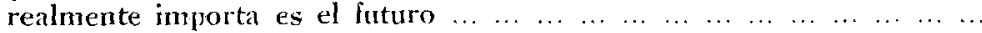

Sólo volviendo a nuestro glorioso y olvidado pasado podremos conseguir un progreso social auténtico $\ldots \begin{array}{lllllllllllll}\ldots & \ldots & \ldots & \ldots & \ldots & \ldots & \ldots & \ldots & \ldots & \ldots & \ldots & \ldots & \ldots\end{array}$

Es preferible no expresar nuestras propias opiniones hasta que hayamos tenido la posibilidad de oir las de aquellos a quienes profesamos un gran respeto

El peor crimen que puede cometer un ser humano es atacar públicamente

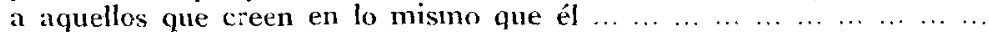

Una persona que se siente atraída por muchas causas o ideales es muy probable que sea un "veleta" o un débil de espíritu ..................

Para conseguir la felicidad futura de la humanidad es necsisario, a veces, tolerar las injusticias del presente $\begin{array}{lllllllllll}\ldots & \ldots & \ldots & \ldots & \ldots & \ldots & \ldots & \ldots & \ldots & \ldots & \ldots\end{array}$

Lo más importante no es el éxito propio, sino el éxito de los híos $\left({ }^{*}\right)$.

A la larga, como mejor se vive es eligiendo amigos y compañeros que

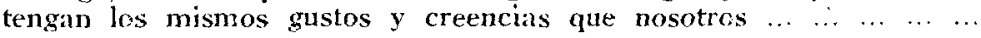

La vida sólo tiene sentido cuando una persona se dedica plenamente a

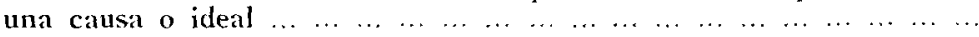

Un g:upo en el que se toleran demasiadas diferencias de opinión entre sus miembros no puede durar mucho tiempo

De todas las filosolías que existen hoy día en el mundo, probablemente

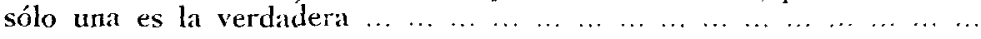

Lo más importante no es tener éxito en este mundo, sino lo que ocurra

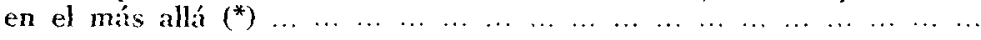

En la historia de la humanidad, se podrian contar con los dedos de la mano el número de pensadores auténticamente geniales $\ldots . . .6 \ldots$

Para que un hombre cumpla su misión en la vida es necesario, a veces,

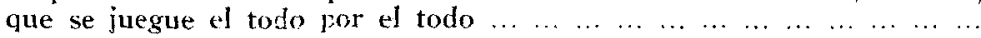

(*) Estos items no fueron utilizados por Rokeach. 


\section{CUADRO II}

Autoritarismo por distintas variables socioeconómicas (*)

\begin{tabular}{c} 
AUTORITARISMO \\
\hdashline Alts
\end{tabular}

\section{OCUPACION DEL PADRE:}

Trabajador no especializado

Trabajador especializado

Independientes

Oficinistas

Cuadros medios

Directivos

$\begin{array}{lll}28 & 24 & (445) \\ 29 & 24 & (345) \\ 30 & 23 & (547) \\ 21 & 31 & (170) \\ 11 & 33 & (150) \\ 12 & 52 & (105)\end{array}$

\section{OCUPACION ENTREVISTADO:}

Trabajador no especializado

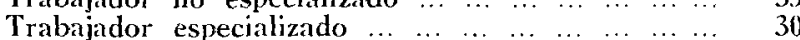

\begin{tabular}{llllllllllllll} 
Independientes & $\ldots$ & $\ldots$ & $\ldots$ & $\ldots$ & $\ldots$ & $\ldots$ & $\ldots$ & $\ldots$ & $\ldots$ & $\ldots$ & $\ldots$ & $\ldots$ & \\
\hline
\end{tabular}

$\begin{array}{lll}35 & 20 & (182) \\ 30 & 23 & (271) \\ 30 & 22 & (207) \\ 19 & 37 & (219) \\ 13 & 37 & (142) \\ 16 & +7 & (51)\end{array}$

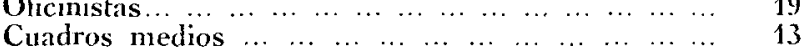

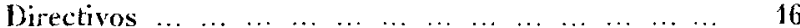

\section{INGRESOS FAMILIARES MENSUALES:}

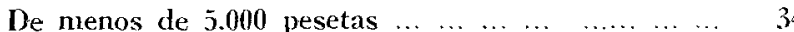

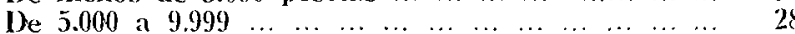

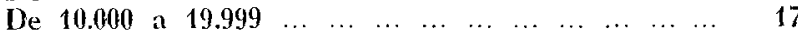

$\begin{array}{lll}34 & 23 & (396) \\ 28 & 23 & (706) \\ 17 & 35 & (376) \\ 11 & 47 & (114)\end{array}$

De 20.0100 a 49.999

NIVEL DE ESTUDIOS:

\begin{tabular}{lllllllllllllll} 
Primarios & $\ldots$ & $\ldots$ & $\ldots$ & $\ldots$ & $\ldots$ & $\ldots$ & $\ldots$ & $\ldots$ & $\ldots$ & $\ldots$ & $\ldots$ & $\ldots$ & $\ldots$ & \\
\hline
\end{tabular}

$\begin{array}{llllllllllllll}\text { Secundarios } & \ldots & \ldots & \ldots & \ldots & \ldots & \ldots & \ldots & \ldots & \ldots & \ldots & \ldots & \ldots & \end{array}$

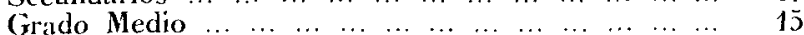

$10 \quad 50$

\section{ASOS DE EDUCACION:}

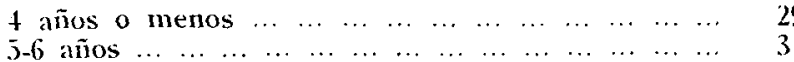

$\begin{array}{llllllllllllllll}7-8 & \text { años } & \ldots & \ldots & \ldots & \ldots & \ldots & \ldots & \ldots & \ldots & \ldots & \ldots & \ldots & \ldots & \ldots & \ldots\end{array}$

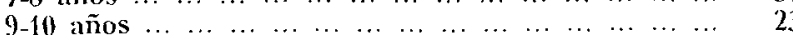

$\begin{array}{lllllllllllllll}11-12 & \operatorname{annos} & \ldots & \ldots & \ldots & \ldots & \ldots & \ldots & \ldots & \ldots & \ldots & \ldots & \ldots & \ldots & \ldots\end{array}$

13 años o más

$\begin{array}{lll}29 & 23 & (366) \\ 31 & 22 & (329) \\ 31 & 18 & (383) \\ 23 & 29 & (251) \\ 17 & 23 & (172) \\ 17 & 43 & (297)\end{array}$

(*) Los porcentajes no suman 100 por no estar incluidos los casos con puntuación "media". 


\section{CUADRO III}

Items de la escala de dogmatismo

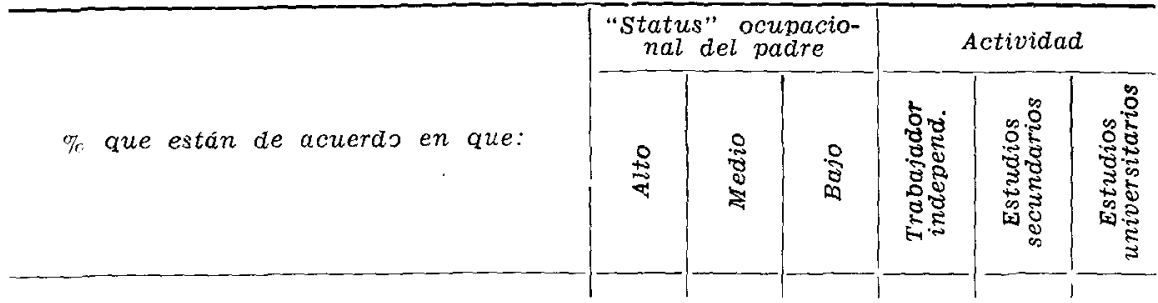

1. De todas las filosofías que existen hoy en el mundo. probablemente sólo una es la verdadera $\ldots \ldots \ldots$... $\ldots . . . .$.

2. El presente está lleno de desgracias e infelicidades casi siempre; Io que realmente importa es el futuro ... ...

3. Llegar a un compromiso con nuestros oponentes resulta peligroso, porque, en muchos casos, nos llevan a traicionar a nuestro propio bando ...

4. Un grupo en el que se toleran dem:siadas diferencias de opinión entre sus miembros no puede durar mucho

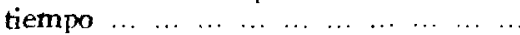

5. Es preferible no expresar muestras pronias opiniones hasta que hayamo: tenido las posibilidades de oir la de aquellos a quienes profesamos un $\begin{array}{llllllllll} & \text { gran respeto } & \ldots & \ldots & \ldots & \ldots & \ldots & \ldots & \ldots & \ldots\end{array}$

6. Sólo volviendo a nuestro glorioso y olvidado pasado podemos conseguir un progreso social auténtico ... ...

7. Una persona que se siente atraída por muchas causas o ideales, es muy probable que sea un "veleta" o un

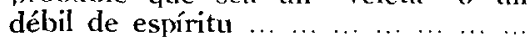

8. Lo más importante no es tener éxito en este mundo, sino lo que ecurra en el más allí

9. La vida sólo tiene scutido cuando una persona se dedica plenamente a una causa o ideal ...

10. En un mundo tan complicado cono el actual, la única fo:ma de enterarse de lo que realmente pasa es at iner:ce a to que nos digan los jefes y exp:tos en quienes podemos confiar

$\begin{array}{llllll}34 & 46 & 46 & 47 & 44 & 35 \\ 27 & 40 & 50 & 49 & 36 & 27 \\ 2.5 & 41 & 43 & 42 & 36 & 24\end{array}$

$\begin{array}{llllll}40 & 52 & 55 & 58 & 51 & 42\end{array}$

$\begin{array}{llllll}45 & 52 & 53 & 53 & 48 & 40\end{array}$

$\begin{array}{llllll}17 & 24 & 33 & 24 & 27 & 7\end{array}$

$\begin{array}{llllll}35 & 48 & 53 & 50 & 46 & 34\end{array}$

$\begin{array}{llllll}43 & 54 & 33 & 51 & 51 & 47\end{array}$

$\begin{array}{llllll}47 & 61 & 57 & 60 & 59 & 62\end{array}$

$$
\mathrm{N}=\quad(213) \quad(945) \quad(701) \quad(381)
$$




\section{CUADRO IV}

\section{Items de la escala " $F$ "}

\begin{tabular}{|c|c|c|c|c|c|c|c|}
\hline \multirow[b]{2}{*}{$\%$ que } & \multirow[b]{2}{*}{ están de acuerdo en que: } & \multicolumn{3}{|c|}{$\begin{array}{l}\text { "Stutus" ocupacio- } \\
\text { nul del padre }\end{array}$} & \multicolumn{3}{|c|}{ Actividad } \\
\hline & & 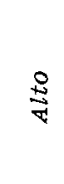 & 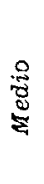 & $\stackrel{\overbrace{}}{\tilde{E}}$ & 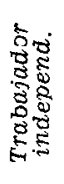 & 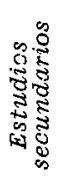 & 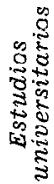 \\
\hline
\end{tabular}

1. En definitiva, las personas se dividen

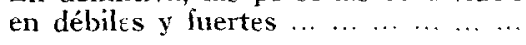

2. Más que leyes, instituciones y prog ämas políticos, lo que necesitamos es un jefe en quien poder confiar ......

3. La obediencia y el respeto a la autoridad son las primeras virtud.s que deben inculcarse a los niños .........

4. Fl industrial y el negociante benciician más a la sociedad que los profesores, investigadores $y$ artistas ...

5. Si se hablara menos $y$ se trabajase más, todo se andaría inejor ... ... ...

$\begin{array}{llllll}36 & 53 & 55 & 52 & 47 & 26\end{array}$

$\begin{array}{llllll}52 & 61 & 66 & 62 & 59 & 47\end{array}$

$\begin{array}{llllll}22 & 30 & 36 & 32 & 27 & 11\end{array}$

$\begin{array}{llllll}56 & 65 & 68 & 67 & 61 & 56\end{array}$

6. Hay que tener fe en la Providencia $y$ obedecer sus decisiones sin vacila-

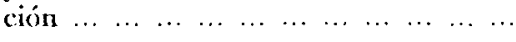

7. Los homosexuales se diferencian bien poco de los criminales y deberían ser tratados como ellos

8. A veces, los jóvenes se sienten rebeldes, pero a medida que envejecen, lo natural es que abandonen sus ideats y se adapten a la realidad ... ... ...

9. Lo que necesita la juventud es disciplina, determinación y deseo de luchar por la patria y la familia ... ...

10. Es nuís importante el orden público que la libertad política ... $\ldots . . . . . .$.

$$
\mathbf{N}=
$$

$\begin{array}{llllll}41 & 53 & 54 & 51 & 51 & 36\end{array}$

$\begin{array}{llllll}27 & 37 & 39 & 35 & 31 & 18\end{array}$

$\begin{array}{llllll}46 & 56 & 58 & 58 & 50 & 47\end{array}$

$\begin{array}{llllll}46 & 57 & 62 & 60 & 53 & 39\end{array}$

$\begin{array}{llllll}4 & 52 & 52 & 49 & 48 & 30\end{array}$

$\begin{array}{llllll}(123) & (945) & (701) & (381) & (228) & (118)\end{array}$ 
Por ctra parte, diez de los items del Cuadro I, y otros diez de la escala "F" fueron incluidos en un cuestionario aplicado a una muestra nacicnal representativa de la población española comprendida entre los 15: y $\operatorname{los} 29^{\circ}$ años. Este estudio fue también realizado por el Instituto de la Opinión Pública en colaboración con el Instituto de la Juventud. Los porcentajes "de acicr 10 " en cada uno de los items relacionado con el "status" ocupacicnal del padre del entre:istado, así como con el sector de actividad de éste, aparecen en los Cuadros III y IV.

\section{Interpretación}

Los resultados de los estudios a que nos hemos referido son coherentes. En ambos aparecen relaciones definidas entre variables sociosconómicas -que suelen adoptarse como indicadcres de clase social- y autoritarismo, medide por las respuestas a items de la escala de dogmatismo de Rokeach y de la escala "F". Estas relaciones apoyan, con muestras españolas, la hipótesis de Lipset del relativo mayor autoritarismo de las clases trabajadoras.

Ahora bien, tal planteamiento no deja de ser cuestionable, tanto desde un punto de vista teórico como metodológico.

En primer lugar, el sutil cambio de acento desde una perspectiva funcional a una perspectiva cognitiva en la teoría de las actitudes implícita en el análisis de Lipset desvirtúa, a mi juicio, el significado original del concepto personalidad autoritaria. Ciertamente que cabe hablar de analogías formales entre el "autoritarismo ideológico" y el "autoritarismo utópico". Ahora bien, en la medida en que ambos entrañan un sistema coherente de creencias -políticas, entre ctras - sobre la realidad, la preponderancia en el entcrne simbólico del primero interfiere en el grado de cristalización del segundo, impidiendo esa compacta pseudocoherencia de todo sistema cognitivo dogmático. El autoritarismo utópico no encuentra el esfuerzo normativo-institucional consustancial con la existencia del primero, y aunque determinadas situaciones, socialmente estructuradas, deberían tender a producirlo en las orientaciones de las personas que en ellas se encuentran, el hecho es que no ccurre de ese modo. Por el contrario, y como hace notar Converse (18), lo que caracteriza el sistema de creencias políticas de las clases bajas es precisamente su falta de consistencia, lógica y psico-lógica. Tal sstructura creencial -o mejor falta de ella- puede cristalizar en autoritarismo litópico, pero no en el vacío, sino en relación dialéctica con un

(18) Converse, Ph.: The Nature of Belief Systems in Mass Publics. Survey Research Center. Universidad de Michigan, Mimeografiado. 
autoritarismo ideológico ya cristalizado. Sin una base motivacicnal que lo active, el potencial antidemocrático de la clase trabajadora inferido desde un sistema cognitivo más restringido es simplemente eso: posibilidad; del mismo modo que el potencial democrático de las clases medias y altas es, claro está, también una pasibiiliáad. Una especificación de las condiciones concretas en que tenderán a realizarse esas posibilidades hace necesario, a mi juicio, una consideración detenida de la estructura motivacional do una y cotras. Una psicología política diferencial de las clases sociales tiene que ser una "psicología de intereses" (Mannheim), que permita una articulación sistemática con una "sociología de intereses". Esta perspectiva nos remitiría no sólo a los determinantes intraculturales específicos del supuesto potencial antidemocrático de la clase trabajadora, sino también a los factores provinientes de su interacción con otras clases scciales.

En segundo lugar, y en relación con lo anterior, tal y como la hipótesis aparece formulada en el IV capítulo del libro de Lipset, las diferencias cualitativas no son debidamente consideradas. No creemos que ni psicológica ni sociopolíticamente habłando la cuestión sea sólo, ni la más importante, la de un más o menos. Determinadas actitudes tienen muy distinta significación para la mentalidad y el comportamiento según la naturaleza de otras axtitudes con las que se encuentran relacionadas. Estar de acuerdo en que "en lin mundo tan complicado como el actual la única manera de enterarse de lo que realmente pasa es atenerse a lo que nos digan los jefes y expertos en quienes podamos confiar" puede suponer, efectivamente, un componente autoritario en porsonas de clase media o alta, pero en el caso de un trabajador puede sencillamente ser la constatación de un hecho de su situación personal y colectiva (19). El que la preocupación por las libertades formales no sea un e'emento constitutivo consciente de la psicología de la clase trabajadora (20) no quiere decir que sea más autoritaria, sino más bien que desde su situación objetiva de clase estas libertades se presentan en principio como no realizadas, como distantes y abstractas, ccmo irrelevantes para su vivir cotidiano. No son experimentadas como necesidad inmediata porque otras necesidades más apremiantes ocupan su campo psicosociclógico. Ahora bien, esas necesidades inherentes a la situación de la clase trabajadcra se configuran en virtud de la específica relación de subordinación con que se encuentra resfecto de otras clases sociales. Esa relación

(19) En este sentido, S. M. Miller y F. Riessman, afirman: "El deseo experimentado por los trabajadores de estructuración, organización y liderazgo eficaz es confundido con un deseo de sumisión". "Working-Class Authoritarianism: A critique of Lipset", en British Journal of Sociology. Vol. 12, 1961. Pág. 269.

(20) Goldmann, L.: Investigaciones dialécticas. Universidad Central de Venezuela, Caracas, 1962. Pág. 102. 
en la medida en que asigna a la clase trabajadora determinadas condiciones reales de existencia, está prefigurando una conciencia cuyos conteniłos no sólo son clantitativa, sino cualitativamente distintes (21). Por otra parte, y aún admitiendo hipotéticamente el mayor autoritarismo de las clases trabajadoras, existe en el planteamiento de Lipset el supuesto y el temor de una rápida correspondencia entre variables intervinientes y compcrtamiento, en este caso comportamiento político. Pero esta correspondencia no siempre se produce, y mucho menos en el nivel del comportamiento colectivo. Toda una serie de variables psicológicas y estructurales mediatizan el supuesto autoritarismo latente y su posible expresión individual y colectiva.

Conviene, en este punto, añadir también algunas observaciones sobre la variable educación, sobre la que, acertadamente, a mi juicio, Ramírez Giménez, en su trabajo ya citado, insiste en repetidas ccasiones. La asociación entre nivel de educación y autoritarismo ha sido puesta de relieve al tiempo mismo de la aparición de "La Personalidad Autoritaria". En los datos que aparecen en este trabajo también puede observarse. Recientemente Lipsitz, reanalizando las diferencias observadas en autoritarismo entre las clases trabajadora y media en una serie de estudios, ha podido constatar que tales diferencias desaparecen cuando se controla por años de educación -incluso, en los items más directamente relacionados con cuestiones políticas y económicas, lcs trabajadores aparecen como menos autoritarios (22).

Estos resultados sugieren que las diferencias observadas en "autoritarismo", medido por la escala " $F$ " $e$ instrumentos similares, entre las distintas clases sociales, no se deben a diferencias reales en esta variable, tal y como teórico-conceptualmente fue definida originariamente, sino más bien a diferencias de complejidad cognoscitiva ( 22 a).

Desde un ángulo metodológico, y de acuerdo con las anteriores observaciones, lo que aparece como realmente problemático y crucial es la validez de instrumentos como la escala "F" y la escala de Rokeach cuando son utilizados para obtener mediciones comparables entre distintas clases sociales. En nuestro caso, además, hay que contar con las diferencias de contexto

(21) En este sentido creo que hay que interpretar la matización de R`míRez. Crisénfz cuando afirma: "Quizás la tesis de Lipset quedaría meior formulada en al sentido de quie, en caso de producirse, la tendencia autoritaria de la clase trabaiadora se traduce en alianza con comunismo, al igual que, en caso de producirse, la tendencia autoritaria de la clase media se traduce en fascismo o totalitarismo". M. Ramírez Giménez, op. cit., pág. 114.

(22) Lipsitz, L.: "Working-Class Authoritarianism: A Re-evaluation". American Sociological Review. Vol. 30, n. ${ }^{\circ}$ 1, 1965. Prass. 103-109.

(22 a) Investigaciones recientes sobre las diferencias lingüísticas de clase ponen de manifiesto, a mi entender, esas diferencias de complejidad cognoscitiva. 
cultural. Las limitaciones señaladas por Miller y Riessman en este sentido me parecen acertadas (23). La observación genérica de Deutscher es probable que haya que considerarla seriamente en este contexto: "La idea de definición crerativa es un mecanismo precisamente diseñado para eliminat el rroblema de la validez. Cuando definimos que el objeto de nuestro interés es lo que miden nuestros instrumentos, no necesitamos preccuparnos ya de su validez" (24). En este sentido, si bien creemos que los datos presentados son fiables, ¿Fudemos decir lo mismo respecto a sı validez? La respuesta se desprende claramente de mis observaciones a la hipótesis de Lipset. Sin embargo, más detenidas investigaciones son necesarias para poder dar una respuesta más concluyente, de la que, desgraciadamente, no nos es posible ofrecer ahora dada la específica estructura de los datos a nuestra disposición.

En cualquier caso, no deja de ser una estimulante paradoja el que la clase trabajadora que, for su relación subordinada, sufre las consecuencias de orientaciones, mecanismos, estructuras y comportamientos autoritarios, exhiba en nuestras definiciones operativas una puntuación más alta en esta dimensión. Probablemente la sociología del conocimiento podría arrojar mucha luz sobre esta paradoja.

\section{Resumen y conclusión}

Resulta difícil en un problema de esta naturaleza extraer conclusiones, incluso frovisionales. No obstante, dada la significación y alcance de la hipótesis planteada por Lipset, y apuntada la necesidad de considerarla en un contexto comparativo, he creído que podria ser sugestivo plantearla en el contexto español, con unos datos cuya estructura es análoga, en muchos casos, a los aducides por Lipset.

Estos datcs, prima facie, parecen apoyar el relativo mayor autoritarismo de la clase trabajadora frente a las clases medias y altas. No obstante, esta pcsible conclusión es cuestionada scbre la base de las premisas teóricas que entrañan un tal planteamiento, sobre todo al cambiar el énfasis desde los aspectos motivacionales y funcionales del autoritarismo a los aspectos cog. nitivos. Tal cambio de acento es considerado como una desvirtuación del concepto original de personalidad autoritaria. Se sugiere también la necesidad de un planteamiento de la hipótesis no sólo en términos cuantitativos sino también cualitativos. Igualmente, y de acuerdo con las observaciones

(23) Mulez y Riessman, op. cit., págs. 267-269.

(24) Itrurscher, 1.: "Looking Backward: Case Studies on the Progress of Metho. dology in Sociological Research". The American Sociologist. Vol. 4, n. ${ }^{\circ}$ 1. $196 \%$. Paigina 35. 
de Miller y de Riessman, se pone en dirda la validez de instrumentos como la escala "F" para establecer comparaciones entre distintas clases sociales. Las diferencias cbservadas en los datos son atribuidas, provisionalmente, a diferencias de complejidad ccznoscitiva entre las clases trabajadora y media y alta. Son reconocidas, sin embargo, las limitaciones de los datos aportados fara fundamentar tales consideraciones, aunque se sugiere su contradicción con el análisis, por cbscrvación participante, de la estructira de las relaciones cotidianas entre las clases en un nivel interpersonal y organizativo. 